\title{
Enzalutamide is superior to bicalutamide for mCRPC
}

Androgen-deprivation therapy (ADT) with enzalutamide is a mainstay of the treatment of patients with metastatic castration-resistant prostate cancer (mCRPC); whereas bicalutamide, another antiandrogen, is widely used in combination with luteinizing hormone-releasing hormone (LHRH) analogues in patients with hormone-treatment naive cancer. However, data on use of bicalutamide in the castration-resistant setting are limited. Now, results of a randomized, double blind, phase II trial confirm that enzalutamide is superior to bicalutamide as a treatment of mCRPC.

375 patients with mCRPC were randomly assigned to receive either enzalutamide or bicalutamide, with concurrent use of LHRH analogues, bisphosphonates or denosumab also permitted throughout the study duration. Treatment was continued until the emergence of evidence of disease progression. Patients in the enzalutamide group had significantly improved progression-free survival (PFS) durations compared with those in the bicalutamide group (15.7 versus 5.8 months; $P<0.0001$ ). Patients receiving enzalutamide had a higher risk of adverse events leading to treatment discontinuation (28\% versus $23 \%$ of the study cohort, respectively), and a similar risk of grade $\geq 3$ adverse events ( $40 \%$ versus $38 \%$ of the study cohort, respectively). Authors also noted that the use of a variety of definitions of disease progression, including a change to antineoplastic therapy or the emergence of skeletal-related adverse events probably better reflects the realities of clinical practice as opposed to relying on radiographically confirmed disease progression as an indicator.

Taken together, data from this trial provide robust evidence that enzalutamide is superior to bicalutamide as ADT in patients with $\mathrm{MCRPC}$, as demonstrated by the delayed disease progression of patients receiving enzalutamide.

Peter Sidaway

ORIGINAL ARTICLE Shore, N. D. Efficacy and safety of enzalutamide versus bicalutamide for patients with metastatic prostate cancer (TERRAIN): a randomised, double-blind, phase 2 study. Lancet Oncol. http://dx.doi.org/10.1016/ S1470-2045(15)00518-5 (2016) 